\title{
2296. Computational approaches to vibration analysis of shells under different boundary conditions - a literature review
}

\author{
Fazl e Ahad', Dongyan Shi², Zarnab Hina ${ }^{3}$ \\ ${ }^{1,2}$ College of Mechanical and Electrical Engineering, Harbin Engineering University, Harbin, China \\ ${ }^{3}$ College of Information and Communication Engineering, Harbin Engineering University, Harbin, China \\ ${ }^{1}$ Corresponding author \\ E-mail: 19had_khattak@hotmail.com, ${ }^{2}$ shidongyan@hrbeu.edu.cn, ${ }^{3}$ zarnabhina@gmail.com
}

Received 1 February 2016; received in revised form 3 August 2016; accepted 17 August 2016

DOI https://doi.org/10.21595/jve.2016.16884

\begin{abstract}
Shells are important structural elements widely used in various engineering applications ranging from outer space to deep oceans such as rockets, aircrafts, missiles, submarines and automobiles etc. A huge amount of research efforts has been devoted to vibration analysis and dynamic behaviors of the shells. Furthermore, a large variety of shell theories and computational methods have been proposed and developed by researchers. For different cases different computational approaches have been used in literature to study the vibration characteristics of shells. This review is aimed to provide contemporarily relevant survey of papers on vibrational characteristics of shells and identification of various methods and approaches that have been used to study its vibration characteristics. Focus has been kept to important and prominent studies and its compilation in a single paper to help future researchers to identify relevant literature quickly and easily and also help them to apply these approaches to study vibration characteristics of other built up and coupled structures.
\end{abstract}

Keywords: vibrations, shells, natural frequency, mode shapes, arbitrary boundary conditions.

\section{Introduction}

Cylindrical shells are important structural elements widely used in various engineering applications ranging from outer space to deep oceans such as rockets, aircrafts, missiles, submarines and automobiles etc. In these applications the shell structures may encounter vibrations and as a result may fail due to material fatigue. Many calamitous incidents have been recorded in the history due to this destructive nature of vibration. Furthermore, these vibrations result in noise which always causes annoyance, discomfort and loss of efficiency to human beings. Therefore, it is of prime importance to completely understand the structural vibrations of shells and reduce them through proper design to ensure a reliable, safe, efficient and lasting structural performance.

An important step in the vibration analysis of shell structures is the evaluation of its vibration characteristics which includes natural frequencies and mode shapes. This modal information plays a key role in the design and vibrations suppression of these structures when subjected to dynamic loads or excitations. Besides various other geometric parameters such as thickness to length ratio, thickness to radius ratio and subtended angle also plays important role in the vibrations, acoustics and safety analysis of these shell structures. Shell vibration problems deal with two main concepts: shell theories and computational approaches. In the last decades, a huge amount of research efforts has been devoted to vibration analysis and dynamic behaviors of the shells and a lager variety of shell theories and computational methods have been proposed and developed by researchers. A comprehensive review on these can be found in Leissa's monograph [1]. This book includes comprehensive results of free vibration frequencies and mode shapes for of shells subjected to different boundary conditions as well as detailed analysis of various shell theories.

As far as the shell deformation theories are concerned, they can be divided into two-dimensional (2-D) theories and three-dimensional (3-D) theory known as 3-D elasticity theory. The two-dimensional theories can be further classified into Classical Shell Theory (CST), 
first order shear deformation theory (FSDT) and higher order shear deformation theory (HSDT). For vibration analysis of thin shells, classical shell theory has been used by many researchers [2-8]. Since the effect of shear deformation in the thickness direction is ignored in such shell structures therefore only proper low frequency results can be obtained by using CST. In order to incorporate the transverse shear stresses through the shell thickness, various types of first order shear deformation theories (FSDTs) were developed for moderately thick shells [9-10]. In these FSDT's the transverse shear strain was assumed to be constant throughout in the thickness direction which resulted in incorporation of a constant shear correction factor to adjust transverse shear stiffness. To overcome the deficiency of FSDT's and further improve the dynamic analysis of moderately thick shell, a number of HSDT's with varying degree of refinements of the kinematics of deformation were developed [11-12]. In these HSDTs the transverse normal stress and strains were ignored resulting in inapplicability of these theories to vibration analysis of thick shells. Later to account for the transverse normal stress and strain components, 3-D elasticity theory was established which is the best choice for analyzing three dimensional thick shells.

Besides development of various shell theories, many accurate and efficient computational methods were also developed to accurately predict the vibration response of shell structures. These computational methods include Ritz method, differential quadrature method (DQM), Galerkin method, wave propagation approach, multiquadric radial basis function method (MRBFM), meshless method, finite element method (FEM), Finite Difference (FD) and discrete singular convolution approach (DSC), etc. These methods, however, have respective drawbacks in that they may be too complex mathematically for routine engineering analysis, be relatively easy to use but limited to special cases, need initial trial functions, or require large amounts of computational effort and consequently high cost. For instance, in a large number of problems, reasonably approximate solutions are desired at only a few specific points in the physical domain. However, in order to get results even at or around a point of interest with acceptable accuracy, traditional FE and FD methods still require the use of a large number of grid points. Consequently, the requirements for CPU time and storage are often unnecessarily large in such cases. On the other hand, the Rayleigh-Ritz and Galerkin methods require less computational effort as compared with the FE and FD methods. However, these methods require one to select initial trial functions satisfying boundary conditions for problems considered, while this is not an easy task in practice. In addition, these methods also need more strenuous formulation effort. Only in some simple cases or problems, these analytical computational methods give the exact solution to a problem. The term "exact solution" means a solution that not only satisfies the governing equation of motion on a point wise basis but also satisfy the boundary conditions.

For the reasons mentioned above different computational approaches have been used in literature for different shell cases to precisely and accurately evaluate vibration characteristics. This literature survey is aimed to compile these different computational approaches in a single paper. In the following text effort has been out to highlight up to date key research work done on the vibration characteristics of shell structures using different computational methods.

\section{Literature review}

A comprehensive review on shell structures can be found in Leissa's 1973 monograph which includes comprehensive results of free vibration frequencies and mode shapes for shells subjected to different boundary conditions. In 1984, Reddy et al. [13] presented exact solution for moderately thick laminated shells by extending Sanders shell theory for doubly curved shells to a shear deformation theory of laminated shells. Exact solution was presented for simply supported, doubly curved, cross-ply laminated shells under sinusoidal, uniformly distributed and concentrated point load at the center. Bench mark exact solution was presented for comparison with other approximate methods like Finite element method and finite difference method. S. K Qatu [14] in his research studied free vibration and static analysis of laminated composite shallow shells and exact solution was obtained for a limited set of boundary conditions and 
lamination sequences. Using the Ritz method, he developed a new approximate procedure to study both the free vibration and static problem.

In another important research on vibrations of cylindrical shallow shells, Lim et al. [15] proposed a higher order shear deformation theory to analyze the effect of various shell geometries and boundary conditions on the vibration responses. A set of orthogonally generated two-dimensional polynomial functions were used to approximate the deflections and rotations of the shells and energy functional based on the principle of extremum energy was employed to obtain governing Eigen matrix equations. Later Lei et al. [16] presented explicit fundamental solutions of the shallow spherical shells involving transverse shear deformation using complex Bessel functions.

K. M. Liew et al. [17] employed Ritz extremum energy principle to study the vibration response of doubly- curved rectangular shells. A refined first order shear deformation theory was developed by considering Lame' parameters for transverse shear strain through the shell thickness which was neglected by earlier researchers. This inclusion resulted in linear transverse shear strain distribution functions instead of constant value. The excellent performance of the computational methodology was illustrated through convergence and comparison studies.

Different boundary value problems lead to the use of different analytical methods. This was studied by Yu et al. [18] in their research on free vibration analysis of open circular cylindrical shells. They presented three analytical methods, generalized Navier method, the generalized Levy method and the method of superposition for free vibration analysis of open circular cylindrical shells with arbitrary combinations of simple boundary conditions. They concluded that only 13 basic shells need to be investigated in order to complete the free vibration analysis of any open circular cylindrical shell. Xi et al. [19] used a semi analytical approach to predict the free vibration characteristics of composite shells of revolution. They considered both the transverse shear deformation and the coupling between symmetric and asymmetric modes in laminated composite shells. They studied the effect of shear deformation, circumferential wave number, thickness to radius ratio, ply angle and stacking sequence on vibration characteristics in detail.

In another important research Lim et al. [20] employed 3-D elasticity approach to study the free vibration of open cylindrical shells. Three-dimensional displacement based extremum energy principle was adopted in their research to obtain the natural frequencies and the corresponding mode shapes. In their research the two-dimensional p-Ritz admissible functions previously used in thick shallow shell studies were generalized to three-dimensional functions by associating the two-dimensional functions with a one-dimensional p-Ritz admissible function. N. M. Price et al. [21] investigated the vibration of cylindrical pipes using Donnell's theory, Love's theory and an improved theory which included the effect of shear deformation and rotary inertia. The results were compared with finite element models of the pipe. Most of the complex three dimensional problems are analyzed by converting it to two dimensional problems through certain assumptions. A similar approach was used by J. Awrejcewicz and V. A. Krysco. In their research they used three-dimensional theory for plates and shells vibrations to estimate a validity of the two dimensional theories application range [22].

Rayleigh Ritz method is considered to be the best choice to obtain Eigen values and corresponding mode shapes. Rayleigh method depends upon the selection of suitable admissible displacement functions. The selection of proper admissible function in the Rayleigh Ritz method is of critical importance. An important research regarding the systematic choice of admissible functions in the Rayleigh Ritz method was conducted by Amabili et al. [23]. They proposed the selection of Eigen functions, of the closest and simpler problem extracted from the one under consideration, as the admissible function. H. Hassis [24], in his research, extended the wrapping high order theory of plate deformation to shells which takes into account the effect of transverse shear deformation and a non-linear distribution of the in-surface displacements with respect to the thickness coordinate. He concluded that for problems which involve rapidly fluctuating loads with the characteristic length of the order of the thickness, or high frequencies analysis of plates and shells, a high order theory is required to give meaningful results. 
On the basis of three-dimensional theory of elasticity, an approximate analysis using a layer wise approach was presented by Loy et al. [25] to study the vibration characteristics of thick circular cylindrical shells. This approach was similar to Finite strip method in a way that thick cylindrical shell was discretized into an arbitrary number of thin cylindrical layers in the thickness direction and each layer was assumed to have a three-dimensional stress state. The displacement functions were approximated by trigonometric functions in the axial and circumferential directions and by some linear shape functions in the radial direction. Extensive results with high accuracy in comparison with the results available in the literature were obtained. In another research Zenkour et al. [26] employed Hamilton-Reissner's hybrid variational approach to deduce a refined first order theory of composite laminated cylindrical shells with non-homogeneous elastic properties. The governing equations were derived without taking into account the shear correction factor. They concluded that the inclusion of non-homogeneity is required for predicting the actual structural response of shells. Lei Zhang [27] in his research on vibrations of shells and plates studied analytical and numerical development on vibrations of shells. He concluded that state-space method and local adaptive differential quadrature methods (LaDQM) are potential powerful methods for the analysis of plates and shells.

P. Ribeiro [28] in his research derived a p-version, hierarchical finite element for doubly curved, moderately thick, isotropic shallow shells and geometrically non-linear free vibrations were investigated. He employed principle of virtual work and d'Alembert's principle to obtain equations of motion which were further solved by predictor-corrector method. With the proposed element low dimensional accurate models were obtained in his research. It was also shown that the element proposed requires far fewer degrees of freedom for accuracy than h-version finite elements. This resulted in a much smaller computational effort when solving the non-linear equations of motion by a continuation method.

A numerical method based on Rayleigh-Ritz method was presented by S. Kandasamy et al. [29] for the forced vibration of open cylindrical shells. A polynomial-type (p-type) displacement method and the state-space modeling approach were used. Three-dimensional strain-displacement relations were used to derive the governing equations and the transient response of the shell with and without damping was sought by transforming the equations to state-space model and then the state-space differential equations were solved using the Runge-Kutta algorithm. C. Adam [30], in his research, addressed nonlinear flexural vibrations of shallow shells composed of three thick layers with different shear flexibility symmetrically arranged with respect to the middle surface. First order shear deformation theory was employed layer wise to obtain the kinematic relations and modified Berger's theory was employed to model the nonlinear characteristics of the structural response.

Ömer Civalek [31], employed Love's first approximation thin shell theory and discrete singular convolution (DSC) method to study the free vibration analysis of laminated conical and cylindrical shell. Free vibrations characteristics were presented graphically for different geometric and material parameters. He concluded that DSC method provides an accurate, efficient means of solving problems for orthotropic laminated conical and cylindrical shells. In another study Civalek et al. employed the same DSC method to study the free vibration analysis of rotating cylindrical shells. The formulations were based on Love's first approximation shell theory and included the effects of initial hoop tension and centrifugal and Coriolis accelerations due to rotation. Frequencies were obtained for different types of boundary conditions and geometric parameters [32].

P. Ribeiro [33], in another research used p-version, hierarchical finite element method to study the forced, large amplitude, periodic vibrations of open, linear elastic and isotropic, cylindrical shells. A shooting method was applied in conjunction with the Newton method to solve the equations of motion. Due to reduced number of approximations and reasonable computational cost, the proposed method is considered to be a good alternative to analyses geometrically non-linear periodic vibrations of shells. Later Ribeiro studied the validity of different approximations in the analysis of geometrically non-linear vibrations of open, cylindrical, 
laminated, fully clamped shells and studied the influence of membrane inertia and shear deformation [34]. In another similar study a generalized differential quadrature method was employed by Tornabene et al. to study the dynamic behavior of functionally graded conical, cylindrical shell and annular plate structure [35]. The first order shear deformation theory was used to obtain the strain displacement relations. A very fast convergence and numerical stability of the method was observed in the results obtained. It was concluded after comparison with FEM codes that the GDQ gives accurate and computationally low cost results for all such structures. Later in another study Tornabene proposed a generalized power law distribution and employed the same GDQ method and studied the dynamic behavior of functionally graded conical, cylindrical shell and annular plate structure. It was concluded that frequency vibration of functionally graded shells and panels of revolution depends on the type of vibration mode, thickness, power-law distribution, power-law exponent and the curvature of the structure [36].

Yusuke Mochida [37], in his dissertation investigated the capability of the Superposition method to obtain the natural frequencies for thin doubly curved shallow shells and also studied the transient response of plates. Due to unavailability of exact solution for the doubly curved shells, he used series of sine and cosine function as displacement functions generated using Galerkin's method. The prescribed boundary conditions of the shell were then satisfied using the Superposition Method. Excellent convergence and agreement was observed with the published results obtained using Rayleigh-Ritz method.

Dr. N. H. Hadi et al. [38] used higher order shear deformation theory to present a nonlinear finite element model for geometrically large amplitude free vibration intact shallow cylindrical and delamination shell analysis. The nonlinearity was introduced using the Green-Lagrange sense and governing equation of the vibrated shell was derived using the Variational approach. They developed a dynamic analysis method to investigate and characterize embedded delamination on the dynamic response of composite laminated structures. Khalili et al. [39], in their research presented closed-form formulation of three-dimensional (3-D) refined higher-order shear deformation theory to study the free vibration analysis of homogenous isotropic circular cylindrical shells subjected to classical boundary conditions. This RHOST accounted for effects of in-plane and rotary inertias as well as the effects of transverse normal and shear strains on the dynamic response of cylindrical shells. The equations of motion were obtained using Hamilton's principle and solutions were obtained using the Galerkin method. Excellent agreement between the results obtained using the refined higher order shear deformation theory and 3-D elasticity theory was observed.

Dai et al. [40], presented a unified analytical method for vibration analysis of circular cylindrical shells with arbitrary boundary conditions including the classical ones. The flexural and in-plane displacements were generally sought, regardless of the boundary conditions, as a simple Fourier series supplemented by several closed-form functions. Rayleigh-Ritz method was employed to find the Eigen values, Eigen vectors and corresponding mode shapes. The beauty of this method is it does not require any changes in solution algorithms to adapt different boundary conditions. Using ten different thin shell theories, Farshidianfar et al. [41], studied the free vibration of circular cylindrical shells subjected to simply supported boundary conditions. The aim of the study was to use the beam function as an approximation for boundary condition to find the natural frequencies of a shell. A good agreement with the experimental results was observed and it was revealed that the approximate method based on Soedel and Kennard-Simplified theories revealed better results compared to other theories.

In another important research, employing the Flugge classical thin shell theory, Zhou et al. [42], studied the free vibrations of cylindrical shells with elastic boundary conditions. The equations of motion for the cylindrical shells were solved by the method of wave propagations. The elastic boundary conditions were achieved using 8 sets of distributed boundary springs. A similar research on circular cylindrical shell with non-uniform elastic restraints was carried out by Yuehua Chen et al. [43]. The exact solution for the problem was obtained using improved Fourier series method, in which each of three displacements of the shell is represented by a Fourier series 
supplemented by several terms introduced to ensure and accelerate the convergence of the series expansions. The unknown expansion coefficients are treated as the generalized coordinates and determined using the Rayleigh-Ritz procedure. It was concluded that for uniform boundary conditions using the improved Fourier series, the results converge very fast however in case of non-uniform elastic restraints the convergence is slow due to the existence of discontinuity in the boundary constraints however the results are still acceptable in practical.

A method previously developed for vibrations of beams and plates was extended by Dai et al. [44] to study the free vibration analysis of circular cylindrical shells with arbitrary elastically restrained boundary conditions. Based on Flügge's theory, an exact series solution was obtained. All the displacements were expressed as the combination of a two dimensional and a one-dimensional Fourier series. One of the important advantages of this approach is that it can be universally applied to shells with a variety of different boundary conditions, without the need of making any corresponding modifications to the solution algorithms and implementation procedures as typically required in other techniques.

Jin et al. [45], presented a unified analytical method based on the first order shear deformation theory to study the vibration analysis of moderately thick composite laminated cylindrical shells subjected to general boundary conditions and arbitrary intermediate ring supports, and various lamination schemes. An exact solution was obtained by using Rayleigh-Ritz procedure based on the energy functions of the shell. Bench mark results were presented for future computational techniques. In another similar study Jin et al. [46], employed Ray-Leigh Ritz method to obtain the exact solution, based on energy functions, for the free vibration analysis of composite laminated cylindrical shells with general elastically restrained boundaries and arbitrary lamination schemes. The displacement functions were presented in the form of standard Fourier cosine series supplemented with auxiliary functions introduced to eliminate all the relevant discontinuities with the displacement and its derivatives at the edges and accelerate the convergence of series representations. New results for different lamination schemes with elastically restrained edges were presented.

$\mathrm{Qu}$ et al. [47], presented an efficient domain decomposition method for solving the free, harmonic and transient vibrations of isotropic and composite cylindrical shells subjected to various combinations of classical and non-classical boundary conditions. The continuity constraints on the segment interfaces were incorporated into the system potential functional by means of a modified variational principle and least-squares weighted residual method. An arbitrarily laminated version of Reissner-Naghdi's shell theory was employed to formulate the theoretical model and double mixed series, i.e., the Fourier series and orthogonal polynomials, were used as admissible displacement functions for each shell segment. The author's concluded that the present approach is an efficient method for the vibration analysis of cylindrical shells owing to its high order of accuracy and small requirement of computational effort. Later Qu et al. [48], derived a general formulation by means of a modified variational principle in conjunction with a multi-segment partitioning procedure on the basis of the first-order shear deformation shell theory to study free, steady-state and transient vibration analyses of functionally graded shells of revolution subjected to arbitrary boundary conditions. Fourier series and different polynomials were applied to expand the displacements and rotations of each shell segment. The versatility of the formulation was demonstrated through the application of the following polynomials: Chebyshev orthogonal polynomials, Legendre orthogonal polynomials, Hermite orthogonal polynomials and power polynomials. Validity and accuracy of the present formulation was confirmed by comparing the solutions with the existing results in the literature and those obtained from finite element analyses.

In another prominent research Qu et al. [49], applied the domain decomposition technique for solving vibration problems of uniform and stepped cylindrical shells with arbitrary boundary conditions. Multilevel partition hierarchy, viz., stepped shell, shell segment and shell domain, was adopted to accommodate the computing requirement of high order vibration modes and responses. The interface continuity constraints were enforced by using a modified variational principle and 
least-squares weighted residual method and the displacement components were expanded in the form Fourier series for the circumferential variable and polynomials/series (i.e., the Chebyshev orthogonal polynomials, Legendre orthogonal polynomials, and simple power series) for the axial variable. The utility and robustness of the method for the application of various basis functions was evaluated. The authors concluded that technique derived is numerically efficient and accurate solution applicable to a wide range of vibration problems of uniform and stepped cylindrical shells.

A variational formulation for predicting the free, steady-state and transient vibrations of composite laminated shells of revolution subjected to various combinations of classical and non-classical boundary conditions were employed by $\mathrm{Qu}$ et al. [50]. A modified variational principle in conjunction with a multi-segment partitioning technique was employed to derive the formulation based on the first-order shear deformation theory. Fourier series and four sets of polynomials, i.e., the Chebyshev orthogonal polynomials of first and second kind, the Legendre orthogonal polynomials of first kind and the ordinary power polynomials were used as admissible functions.

Xie et al. [51], presented a novel and efficient solution by using Haar wavelet discretization method for free vibrations of thin cylindrical shells subjected to various boundary conditions. The Goldenveizer-Novozhilov shell theory was adopted to formulate the theoretical model. The displacements and their derivatives in the governing equations were represented by Haar wavelet series and their integrals in the axial direction and the Fourier series in the circumferential direction. The advantage of this method is its simplicity, fast convergence, low computational cost and high precision. This method can easily be extended to the composite laminated and functionally graded shells subjected to arbitrary boundary conditions.

Based on Donell-Mushtari shell theory Xing et al. [52], developed a semi-analytical differential quadrature finite element method to obtain the exact solution for free vibration of circular cylindrical shells with classical boundary conditions. High accuracy membrane approximation solutions are also presented for free vibration of thin isotropic closed circular cylindrical shell and open circular cylindrical shells with shear diaphragm conditions. A unified and exact solution method was developed for the free vibration analysis of composite laminated shallow shells with general elastic boundary conditions by Ye et al. [53]. Each of the shell displacements, regardless of boundary conditions, was expanded as a standard Fourier cosine series supplemented with closed-form auxiliary functions introduced to eliminate all the relevant discontinuities with the displacement and its derivatives at the edges and accelerate the convergence of series representations. Rayleigh-Ritz procedure was then employed to obtain the exact solution based on the energy functions of the shell.

In another study Ye et al. [54] presented a Chebyshev-Ritz formulation to investigate the vibrations of composite laminated deep open shells with various shell curvatures and arbitrary restraints, including cylindrical, conical and spherical ones. First-order shear deformation shell theory was employed to include the effects of rotary inertias and shear deformation. Regardless of boundary conditions, each of displacements and rotations of the open shells was invariantly expressed as Chebyshev orthogonal polynomials of first kind in both directions which were further solved using the Rayleigh-Ritz procedure based on the energy functional of the open shells. Employing the improved Fourier series method Aiguo et al. [55], studied the natural vibration analysis of orthotropic cylindrical shells. Based on the energy principles, in combination with the Rayleigh-Ritz procedure the system characteristic matrix function was obtained.

Alijani et al. [56], compiled a comprehensive literature review on geometrically non-linear free and forced vibrations of shells made of traditional and advanced materials. Prominent studies from the period 2003 to 2013 were presented on closed shells and curved panels made of isotropic, laminated composite, piezoelectric, functionally graded and hyperelastic materials and great attention was given to non-linear vibrations of shells subjected to normal and in-plane excitations. Reduced-order models and their accuracy including perturbation techniques, proper orthogonal decomposition, non-linear normal modes and meshless methods were reviewed in depth. 
Guoyong Jin et al. [57], presented a simple and efficient solution approach based on Haar wavelet method to study the free vibration analysis of functionally graded (FG) cylindrical shell. First order shear deformation theory was used to formulate the theoretical model. First of all, the using Levi approach was used for separation of variables then Haar wavelet discretization was applied with respect to the axial direction and Fourier series was assumed with respect to the circumferential direction. The constants appearing from the integrating process were determined by boundary conditions, and thus the partial differential equations were transformed into algebraic equations and subsequently the natural frequencies were obtained by solving these algebraic equations. Detailed parametric analysis was carried out to show the effects of some geometrical and material parameters on the natural frequencies of FG cylindrical shells. In another research Jin et al. [58], developed an accurate modified Fourier series solution for free vibration analysis of truncated conical shells with general elastic boundary conditions. The displacement functions were presented as a standard Fourier cosine series supplemented by closed form auxiliary functions to ensure and accelerate the convergence of the series expansion. All the expansion coefficients were treated as the generalized coordinates and determined using the Rayleigh-Ritz method.

In another important research Guoyong Jin et al. [59], developed unified method based on first order shear deformation theory to study the vibrations of various composite laminated structure elements of revolution with general elastic restraints including cylindrical, conical, spherical shells and annular plates. Regardless of boundary conditions, each displacement and rotation component of the structures was invariantly expressed as the superposition of a Fourier cosine series and two supplementary functions. On the basis of energy functional of structure elements, the exact series solutions were obtained using the Rayleigh-Ritz procedure. A variety of new vibration results including frequencies and mode shapes for composite laminated cylindrical, conical, spherical shells and annular plates with classical and elastic restraints as well as different geometric and material parameters were presented.

Jiang et al. [60], studied the free vibration of doubly curved shallow shells reinforced by any number of beams of arbitrary lengths. Distributed elastic restraints were used to specify generally the boundary conditions along the shell edges and the coupling conditions between the shell and its reinforcing beams. Each of the displacements was invariably expressed as a simple trigonometric series with accelerated and uniform convergence over the solution domains of interest. All the unknown expansion coefficients were treated as the generalized coordinates and solved using the Rayleigh-Ritz technique. The authors concluded that the current method provides a unified means for solving a wide range of shell problems involving various practical complications with respect to, for example, the boundary conditions, the coupling conditions, the number of stiffeners, and the lengths and locations of the stiffeners.

Based on Donell type shell deformation theory and employing Galerkin method, Najafov et al. [61], studied the vibration of non-homogeneous orthotropic cylindrical shells, based on the shear deformation theory. The Young's moduli, shear moduli and density of the orthotropic material were assumed as continuous functions of thickness coordinate. Lowest values of non-dimensional frequency parameters for non-homogeneous orthotropic cylindrical shells with and without shear deformation and rotary inertia were obtained and results are verified by comparing the obtained values with those in the existing literature.

$\mathrm{Su}$ et al. [62], presented the free vibration analysis of functionally graded open shells including cylindrical, conical and spherical ones with arbitrary subtended angle and general boundary conditions. Based on first order shear deformation theory, modified Fourier series method along with Rayleigh-Ritz method was employed to derive the formulation. The Fourier series was expressed in the form of linear superposition of double cosine series and auxiliary functions. Some new results of FGM open shells with elastic restraints were presented to serve as benchmark for future research. Later Su et al. [63-64], in similar studies developed unified method based on modified Fourier series approach, for free vibration analysis of functionally graded spherical, cylindrical, conical shells and annular plates with general boundary conditions using the first-order 
shear deformation theory and Rayleigh-Ritz procedure.

Using first order shear deformation theory, Xie et al. [65], employed Haar wavelet discretization method to study the free vibration analysis of composite laminated conical, cylindrical shells and annular plates with various boundary conditions. The equations of motion were derived by applying the Hamilton's principle. The displacement and rotation fields were expressed as products of Fourier series for the circumferential direction and Haar wavelet series and their integral along the meridional direction. In other similar studies they employed the same method and studied the free vibration analysis of functionally graded (FG) conical shells and annular plates and also free vibration analysis of composite laminated cylindrical shells subjected to various boundary conditions $[66,67]$.

Xuan et al. [68], extended the application of finite volume method to structural-acoustic problems. They proposed a three-dimensional time domain finite volume method to predict the transient response and natural characteristics of structural-acoustics coupling systems. The computational domain is divided into structural and acoustic sub-domains and the interaction between them was implemented by particle acceleration continuity condition and traction equilibrium condition. The structural-structural and acoustic-acoustic coupling algorithms were implemented by changing material properties without other special treatments. Numerical examples were presented to discuss the influence of water depth variation on natural characteristics of system. It was found that the increase of water depth causes the enhancement of interaction between solid and fluid and the decrease of structural natural frequency.

Based on three-dimensional theory of elasticity, Ye et al. [69], developed a unified method for vibration analysis of thick cylindrical shells with general end conditions and resting on elastic foundations. A standard Fourier cosine series supplemented with four auxiliary functions were used to represent the displacement functions. An accurate solution was obtained using the energy functions of the shell. Same theory and computational procedure was adopted in another study by Ye et al. [70], to study the free vibration of laminated functionally graded (FG) spherical shells with general boundary conditions and arbitrary geometric parameters. In another similar study Ye et al. studied the free vibration analysis of open shells subjected to arbitrary boundary conditions and various geometric parameters such as subtended angle, conicity using a general classical shell theory in conjunction with Chebyshev polynomials and Rayleigh-Ritz procedure. It was concluded that the derived formulation is general in the sense that it can easily accommodate different boundary conditions and geometric dimensions (shallow or deep), different types of shells (cylindrical, conical or spherical). It also offers a unified operation for the entire restraining conditions and the change of boundary conditions from one case to another is as easy as changing structural parameters without the need of making any change to the solution procedure [71].

In another research on vibration analysis of functionally graded cylindrical shells, Beni et al. [72], used modified couple stress theory in conjunction with first order shear deformation shell theory and based on Hamilton's principle derived general equations of motion for classical and non-classical boundary conditions. Using the Navier procedure, the free vibrations of simply supported functionally graded cylindrical nano shell were obtained, and the effects of parameters such as dimensionless length scale parameter, distribution of FG properties, thickness, and length on the natural frequency were identified and was compared with the classical theory.

Ma et al. [73], employed Fourier-Ritz approach to study the vibration analysis of coupled cylindrical shell and annular plate systems under general boundary and coupling conditions. The displacements of each substructure were invariably expressed as the modified Fourier series composed of a classical Fourier series and auxiliary functions. The auxiliary functions were introduced to remove the potential discontinuities of displacements and their derivatives and accelerate the convergence of the expansion series. Tornabene et al. [74], studied the free vibration analysis of one-layered and multilayered isotropic, composite and sandwich cylindrical and spherical shell panels to compare classical two-dimensional (2D) and three-dimensional (3D) finite elements (FEs), classical and refined 2D generalized differential quadrature (GDQ) methods and an exact three-dimensional solution. Low and high order frequencies were analyzed for thick 
and thin simply supported structures.

In the literature review above, development of various theories as well as large number of computational models proposed to predict the vibration analysis of shells have been discussed in detail. It can be observed that in vibration analysis of shells, a large number of non-polynomial based higher order shear deformation theories have been developed however they have not been as widely used as polynomial based theories. Most of the studies based on the non-polynomial functions are limited to analytical solutions of linear problems. Therefore, the development of numerical models based on the non-polynomial functions is necessary to fully assess the accuracy as well as efficiency of the non-polynomial function based higher shear deformation theories. Furthermore, most of the current studies on the 3D analysis of shells are limited to analytical approaches for geometrically linear bending, buckling and free vibration problems with various combinations of classical boundary conditions (simply supported or clamped) boundary conditions on all edges. However, in practical engineering applications the shell structures are not always subjected to these classical boundary conditions rather they are subjected to elastic boundary conditions. Therefore, accurate prediction of vibration characteristics of shell structures subjected to dynamic loads and general elastic boundary conditions is a challenging task for future researchers.

Moreover, as the application of shell type structures has extended from mechanical engineering or aerospace to other fields like medical engineering or nano engineering, therefore the new application trends demand improvement of the theoretical foundations of shell theories since new effects must be taken into account. For example, in case of small size shell like structures (nano tubes), the surface effect plays an important role in the vibration analysis of these structural elements as the material properties changes significantly with the decrease in size. Furthermore, the damage identification and health monitoring of structural and mechanical systems from the study of changes in their vibration characteristics is also a field of interest for future researchers.

\section{Conclusions}

Various analytical methods and computational approaches used for vibration analysis of shells have been identified and highlighted in this review paper. It is observed that in various analytical methods and computational approaches, a continuous change in solution algorithm is required to accommodate different boundary conditions (classical, elastic, uniform \& non-uniform), geometric dimensions (shallow or deep) and types of shells (cylindrical, conical or spherical) etc. However, the Modified Fourier series method has been observed to be universal for vibration analysis in a way that it can easily accommodate different boundary conditions, geometric dimensions, and different types of shells. Furthermore, it does not require any procedural modification or change in solution algorithm to accommodate these changes and therefore it can easily be applied to other $2 \mathrm{D}$ and $3 \mathrm{D}$ complex built up structures to study their vibration characteristics due to its fast convergence, high accuracy and low computational cost.

\section{Acknowledgements}

The authors gratefully acknowledge the financial support from the National Natural Science Foundation of China (No. U1430236) and Natural Science Foundation of Heilongjiang Province of China (No. E2016024).

\section{References}

[1] Leissa Vibrations of Shells. National Aeronautics and Space Administration, Washington, DC, 1973.

[2] Love A. E. H. The small free vibrations and deformation of a thin elastic shell. Philosophical Transactions of the Royal Society, Vol. 179, 1888, p. 491-546. 
[3] Zhu C., Du H. Free vibration analysis of Laminated composite cylindrical shells by DQM. Composites Part B, Vol. 28, 1997, p. 367-374.

[4] Loy C. T., Lam K. Y., Reddy J. N. Vibration of functionally graded cylindrical shells. International Journal of Mechanical Sciences, Vol. 41, 1999, p. 309-324.

[5] Pradhan S. C., Loy C. T., Lam K. Y., Reddy J. N. Vibration characteristics of functionally graded cylindrical shells under various boundary conditions. Applied Acoustics, Vol. 61, 2000, p. 111-129.

[6] Haddadpour H., Mahmoudkhani S., Navazi H. M. Free vibration analysis of functionally graded cylindrical shells including thermal effects. Thin Walled Structures, Vol. 45, 2007, p. 591-599.

[7] Iqbal Z., Naeem M. N., Sultana N. Vibration characteristics of FGM circular cylindrical shells using wave propagation approach. Acta Mechanica, Vol. 208, 2009, p. 237-248.

[8] Sofiyev A. H. The vibration and stability behavior of freely supported FGM conical shells subjected to external pressure. Composite Structures, Vol. 89, 2009, p. 356-366.

[9] Hajianmaleki M., Qatu M. S. Transverse vibration analysis of generally laminated two-segment composite shafts with a lumped mass using generalized differential quadrature. Journal of Vibration Control, Vol. 19, 2013, p. 2013-2021.

[10] Asadi E., Wang W., Qatu M. S. Static and vibration analyses of thick deep laminated cylindrical shells using 3D and various shear deformation theories. Composite Structures, Vol. 94, 2012, p. 494-500.

[11] Bhimaraddi A. A higher order theory for free vibration analysis of circular cylindrical shells. International Journal of Solid and Structure, Vol. 20, 1984, p. 623-630.

[12] Reddy J. N. On refined computational models of composite laminates. International Journal of Numerical Method in Engineering, Vol. 27, 1989, p. 361-382.

[13] Reddy J. N., Asce M. Exact solution of Moderately thick laminated shells. Journal of Engineering Mechanics, Vol. 110, Issue 5, 1984, p. 794-809.

[14] Khalil Qatu Mohamad-Subhi Free vibrations and Static Analysis of Laminated Composite shallow shells. Ph.D. Dissertation, Ohio State University, 1989.

[15] Lim C. W., Liew K. W. A higher order theory for vibration of shear deformable cylindrical shallow shells. International Journal of Mechanical Sciences, Vol. 37, 1995, p. 277-295.

[16] Lei Xiao-Yan, Huang Mao-Kuang, Wang Xiuxi Analysis of the explicit fundamental solution of a shallow spherical shell involving shear deformation. Applied Mathematical Modelling, Vol. 19, 1995, p. 194-200.

[17] Liew K. M., Lim C. W. A Ritz vibration analysis of doubly-curved rectangular shallow shells using a refined first-order theory. Computer Methods in Applied Mechanics and Engineering, Vol. 127, 1995, p. 145-162.

[18] Yu S. D., Cleghorn W. L., Fenton R. G. On the accurate analysis of free vibrations of Open Circular cylindrical shells. Journal of Sound and Vibration, Vol. 188, 1995, p. 315-336.

[19] Xi Z. C., Yam L. H., Leung T. P. Semi Analytical study of free vibration of composite shell of revolution based on Reissner-Mindlin assumption. International Journal of Solids and Structures, Vol. 33, 1996, p. 851-863.

[20] Lim C. W., Liew K. M., Kitipornchai S. Vibration of open cylindrical shells: a three-dimensional elasticity approach. Journal of Acoustical Society of America, Vol. 104, 1998.

[21] Price N. M., Liu M., Taylor R. Eatock Vibrations of cylindrical pipes band open shells. Journal of Sound and Vibration, Vol. 218, 1998, p. 361-387.

[22] Awrejcewicz J., Krysco V. A. 3-D Theory vs 2-D approximate theory of free orhotropic (isotropic) plate and shell vibrations. Part 2: numerical algorithms and analysis. Journal of Sound and Vibration, Vol. 226, 1999, p. 831-871.

[23] Amabili M., Garziera R. A technique for the systematic choice of admissible functions in the Rayleigh-Ritz method. Journal of Sound and Vibration, Vol. 224, 1999, p. 519-539.

[24] Hassis H. A Wrapping-Kirchoff and a Wrapping-Mindlin theory of shell deformation. Journal of Sound and Vibration, Vol. 225, 1999, p. 633-653.

[25] Loy C. T., Lam K. Y. Vibrations of thick cylinderical shells on the basis of three-dimensional theory of elasticity. Journal of Sound and Vibration, Vol. 226, 1999, p. 719-737.

[26] Zenkour A. M., Fares M. E. Bending, buckling and free vibration of non-homogeneous composite laminated cylindrical shells using a refined first-order theory. Composites Part B, Vol. 32, 2001, p. 237-247.

[27] Zhang Lei Analytical and Numerical Development on Vibrations of Shells. School of Engineering and Industrial Design, University of Western Sydney, 2005. 
[28] Ribeiro Pedro A hierarchical finite element for geometrically non-linear vibration of doubly curved, moderately thick isotropic shallow shells. International Journal for Numerical methods in Engineering, Vol. 56, 2003, p. 715-738.

[29] Kandasamy Selvakumar, Singh Anand V. Transient vibration analysis of open circular cylindrical shells. Journal of Vibration and Acoustics, Vol. 128, Issue 3, 2005, p. 366-374.

[30] Adam Christoph Moderately large vibrations of doubly curved shallow open shells composed of thick layers. Journal of Sound and Vibration, Vol. 299, 2007, p. 854-868.

[31] Civalek Ömer Numerical analysis of free vibrations of laminated composite conical and cylindrical shells: discrete singular convolution (DSC) approach. Journal of Computational and Applied Mathematics, Vol. 205, 2007, p. 251-271.

[32] Civalek Ömer, Gurses Murat Free vibration analysis of rotating cylindrical shells using discrete singular convolution technique. International Journal of Pressure Vessels and Piping, Vol. 86, 2009, p. 677-683.

[33] Ribeiro Pedro Forced large amplitude periodic vibrations of cylindrical shallow shells. Finite Elements in Analysis and Design, Vol. 44, 2008, p. 657-674.

[34] Ribeiro Pedro On the influence of membrane inertia and shear deformation on the geometrically non-linear vibrations of open, cylindrical, laminated clamped shells. Composites Science and Technology, Vol. 69, 2009, p. 176-185.

[35] Tornabene Francesco, Viola Erasmo, Inman Daniel J. 2-D differential quadrature solution for vibration analysis of functionally graded conical, cylindrical shell and annular plate structures. Journal of Sound and Vibration, Vol. 328, 2009, p. 259-290.

[36] Tornabene Francesco Free vibration analysis of functionally graded conical, cylindrical shell and annular plate structures with a four-parameter power-law distribution. Computational Methods in Applied Mechanical Engineering, Vol. 198, 2009, p. 2911-2935.

[37] Mochida Yusuke Free Vibrations of Plates and Shells Using the Superposition Method. The University of Waikato, 2010.

[38] Nabil Hassan Hadi and Kayser Aziz Ameen Nonlinear free vibration of cylindrical shells with delamination using high order shear deformation theory - a finite element approach. American Journal of Scientific and Industrial Research, Vol. 2, 2011, p. 251-277.

[39] Khalili S. M. R., Davar A., Fard K. Malekzadeh Free vibration analysis of homogeneous isotropic circular cylindrical shells based on a new three-dimensional refined higher-order theory. International Journal of Mechanical Sciences, Vol. 56, 2012, p. 1-25.

[40] Dai Lu, Yang Tiejun, Li W. L., Du Jingtao, Jin Guoyong Dynamic analysis of circular cylindrical shells with general boundary conditions using modified Fourier series method. Journal of Vibration and Acoustics, Vol. 134, Issue 5, 2012.

[41] Farshidianfar Anooshiravan, Oliazadeh Pouria Free vibration analysis of circular cylindrical shells: comparison of different shell theories. International Journal of Mechanics and Applications, Vol. 2, 2012, p. 74-80.

[42] Zhou Haijun, Li Wanyou, Lv Binglin, Li W. L. Free vibrations of cylindrical shells with elasticsupport boundary conditions. Applied Acoustics, Vol. 73, 2012, p. 751-756.

[43] Chen Yuehua, Jin Guoyong, Liu Zhigang Free vibration analysis of circular cylindrical shell with non-uniform elastic boundary constraints. International Journal of Mechanical Sciences, Vol. 74, 2013, p. 120-132.

[44] Dai Lu, Yang Tiejun, Du Jingtao, Li W. L., Brennan M. J. An exact series solution for the vibration analysis of cylindrical shells with arbitrary boundary conditions. Applied Acoustics, Vol. 74, 2013, p. 440-449.

[45] Jin Guoyong, Ye Tiangui, Ma Xianglong, Chen Yuehua, Su Zhu, Xie Xiang A unified approach for the vibration analysis of moderately thick composite laminated cylindrical shells with arbitrary boundary conditions. International Journal of Mechanical Sciences, Vol. 75, 2013, p. 357-376.

[46] Jin Guoyong, Ye Tiangui, Chen Yuehua, Su Zhu, Yan Yuquan An exact solution for the free vibration analysis of laminated composite cylindrical shells with general elastic boundary conditions. Composite Structures, Vol. 106, 2013, p. 114-127.

[47] Qu Yegao, Hua Hongxing, Meng Guang A domain decomposition approach for vibration analysis of isotropic and composite cylindrical shells with arbitrary boundaries. Composite Structures, Vol. 95, 2013, p. 307-321. 
[48] Qu Yegao, Long Xinhua, Yuan Guoqing, Meng Guang A unified formulation for vibration analysis of functionally graded shells of revolution with arbitrary boundary conditions. Composites: Part B, Vol. 50, 2013, p. 381-402.

[49] Qu Yegao, Chen Yong, Long Xinhua, Hua Hongxing, Meng Guang Free and forced vibration analysis of uniform and stepped circular cylindrical shells using a domain decomposition method. Applied Acoustics, Vol. 74, 2013, p. 425-439.

[50] Qu Yegao, Long Xinhua, Wu Shihao, Meng Guang A unified formulation for vibration analysis of composite laminated shells of revolution including shear deformation and rotary inertia. Composite Structures, Vol. 98, 2013, p. 169-191.

[51] Xie Xiang, Jin Guoyong, Liu Zhigang Free vibration analysis of cylindrical shells using the Haar wavelet method. International Journal of Mechanical Sciences, Vol. 77, 2013, p. 47-56.

[52] Xing Yufeng, Liu Bo, Xu Tengfei Exact solutions for free vibration of circular cylindrical shells with classical boundary conditions. International Journal of Mechanical Sciences, Vol. 75, 2013, p. $178-188$.

[53] Ye Tiangui, Jin Guoyong, Chen Yuehua, Ma Xianglong, Su Zhu Free vibration analysis of laminated composite shallow shells with general elastic boundaries. Composite Structures, Vol. 106, 2013, p. 470-490.

[54] Ye Tiangui, Jin Guoyong, Su Zhu, Jia Xingzhao A unified Chebyshev-Ritz formulation for vibration analysis of composite laminated deep open shells with arbitrary boundary conditions. Achieve of Applied Mechanics, Vol. 84, 2014, p. 441-471.

[55] Zhang Aiguo, Li Wenda, Du Jingtao, Dai Lu, Yang Tiejun Natural vibration analysis of the orthotropic cylindrical shell structure with various boundary conditions. Journal of Harbin Engineering University, Vol. 35, 2014.

[56] Alijani Farbod, Amabili Marco Non-linear vibrations of shells: a literature review from 2003 to 2013. International Journal of Non-Linear Mechanics, Vol. 58, 2014, p. 233-257.

[57] Jin Guoyong, Xie Xiang, Liu Zhigang The Haar wavelet method for free vibration analysis of functionally graded cylindrical shells based on the shear deformation theory. Composite Structures, Vol. 108, 2014, p. 435-448.

[58] Jin Guoyong, Ma Xianglong, Shi Shuangxia, Ye Tiangui, Liu Zhigang A modified Fourier series solution for vibration analysis of truncated conical shells with general boundary conditions. Applied Acoustics, Vol. 85, 2014, p. 82-96.

[59] Jin Guoyong, Ye Tiangui, Jia Xingzhao, Gao Siyang A general Fourier solution for the vibration analysis of composite laminated structure elements of revolution with general elastic restraints. Composite Structures, Vol. 109, 2014, p. 150-168.

[60] Jiang Shiliang, Li Wen L., Yang Tiejun, Du Jingtao Free vibration analysis of doubly curved shallow shells reinforced by any number of beams with arbitrary lengths. Journal of Vibration and Control, 2014.

[61] Najafov A. M., Sofiyev A. H., Kuruoglu N. Vibration analysis of non-homogeneous orthotropic cylindrical shells including combined effect of shear deformation and rotary inertia. Meccanica, Vol. 49, 2014, p. 2491-2502.

[62] Su Zhu, Jin Guoyong, Ye Tiangui Free vibration analysis of moderately thick functionally graded open shells with general boundary conditions. Composite Structures, 2014.

[63] Su Zhu, Jin Guoyong, Shi Shuangxia, Ye Tiangui A unified accurate solution for vibration analysis of arbitrary functionally graded spherical shell segments with general end restraints. Composite Structures, Vol. 111, 2014, p. 271-284.

[64] Su Zhu, Jin Guoyong, Shi Shuangxia, Ye Tiangui, Jia Xingzhao A unified solution for vibration analysis of functionally graded cylindrical, conical shells and annular plates with general boundary conditions. International Journal of Mechanical Sciences, Vol. 80, 2014, p. 62-80.

[65] Xie Xiang, Jin Guoyong, Li Wanyou, Liu Zhigang A numerical solution for vibration analysis of composite laminated conical, cylindrical shell and annular plate structures. Composite Structures, Vol. 111, 2014, p. 20-30.

[66] Xie Xiang, Jin Guoyong, Ye Tiangui, Liu Zhigang Free vibration analysis of functionally graded conical shells and annular plates using the Haar wavelet method. Applied Acoustics, Vol. 85, 2014, p. $130-142$.

[67] Xie Xiang, Jin Guoyong, Yan Yuquan, Shi S. X., Liu Zhigang Free vibration analysis of composite laminated cylindrical shells using the Haar wavelet method. Composite Structures, Vol. 109, 2014, p. $169-177$. 
[68] Xuan Ling-kuan, Jin Guo-yong, Gong Jing-feng, Zhang Wen-ping, Ming Ping-jian Time domain finite volume method for three-dimensional structural-acoustic coupling analysis. Applied Acoustics, Vol. 76, 2014, p. 138-149.

[69] Ye Tiangui, Jin Guoyong, Shi Shuangxia, Ma Xianglong Three-dimensional free vibration analysis of thick cylindrical shells with general end conditions and resting on elastic foundations. International Journal of Mechanical Sciences, Vol. 84, 2014, p. 120-137.

[70] Ye Tiangui, Jin Guoyong, Su Zhu Three-dimensional vibration analysis of laminated functionally graded spherical shells with general boundary conditions. Composite Structures, Vol. 116, 2014, p. 571-588.

[71] Ye Tiangui, Jin Guoyong, Chen Yuehua, Shi Shuangxia A unified formulation for vibration analysis of open shells with arbitrary boundary conditions. International Journal of Mechanical Sciences, Vol. 81, 2014, p. 42-59.

[72] Tadi Beni Yaghoub, Mehralian Fahimeh, Razavi Hamed Free vibration analysis of size-dependent shear deformable functionally graded cylindrical shell on the basis of modified couple stress theory. Composite Structures, Vol. 120, 2015, p. 65-78.

[73] Ma Xianglong, Jin Guoyong, Shi Shuangxia, Ye Tiangui, Liu Zhigang An analytical method for vibration analysis of cylindrical shells coupled with annular plate under general elastic boundary and coupling conditions. Journal of Vibration and Control, 2015.

[74] Tornabene F., Brischetto S., Fantuzzi N., Viola E. Numerical and exact models for free vibration analysis of cylindrical and spherical shell panels. Composites Part B, Vol. 81, 2015, p. 231-250.

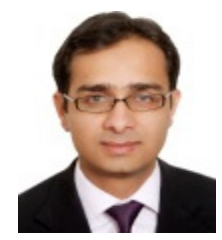

Fazl e Ahad received Master's degree in mechanical engineering design from University of Engineering and Technology Peshawar Pakistan. Presently pursuing Ph.D. from Harbin Engineering University, China in the field of mechanical design and theory. research field includes vibrations of structures.

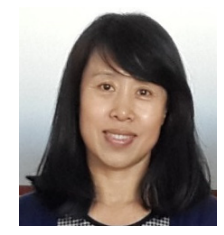

Dongyan Shi is a Professor in School of Mechanical and Electrical Engineering, Harbin Engineering University, China. Her research field includes modern mechanical design theory and method, mechanism and structure strength.

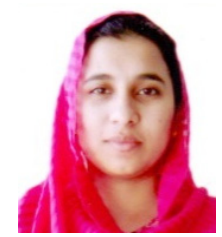

Zarnab Hina completed her Bachelor in Telecommunication Engineering from University of Engineering and Technology Taxila, Pakistan. Presently, she is pursuing Masters in information and communication engineering from Harbin Engineering University, China. Major research interest field includes optical orthogonal frequency division multiplexing. 